\title{
Biochemical and Molecular Biological Studies on Oral Cancer: An Overview
}

\author{
Rashi Srivastava $^{1,2}$, Rolee Sharma ${ }^{2}$, Sanjay Mishra ${ }^{1,3, *}$ and R.B. Singh ${ }^{4}$ \\ ${ }^{I}$ Department of Biotechnology \& Microbiology, IFTM, Delhi Road, Moradabad 244 001, U.P., India \\ ${ }^{2}$ Department of Biotechnology, Integral University, Lucknow 226 015, U.P., India \\ ${ }^{3}$ Department of Biotechnology, College of Engineering \& Technology, IFTM Campus, Delhi Road, Moradabad 244 001, \\ U.P., India \\ ${ }^{4}$ Halberg Hospital and Research Center, Civil Lines, Moradabad 244 001, UP, India
}

\begin{abstract}
Oral cancer is the cancerous tissue growth located in the oral cavity. It may arise as a primary lesion originating in any of the oral tissues, by metastasis from a distant site of origin, or by extension from a neighboring anatomic structure, such as the nasal cavity or the maxillary sinus. Smoking and other tobacco use are associated with about 75 percent of oral cancer cases. Alcohol use is another high-risk activity associated with oral cancer. Infection with human papillomavirus (HPV), particularly type 16 (there are over 120 types), is a known risk factor and independent causative factor for oral cancer. Oncogenes, gain-of-function mutations of highly regulated normal cellular counterparts (proto-oncogenes), are likely involved in the initiation and progression of oral neoplasia. Cellular oncogenes were initially discovered by the ability of tumor cell DNA to induce transformation in gene transfer assays. Mechanisms of activation of these cellular oncogenes include point mutations and DNA re-arrangements. Several of these cellular oncogenes are homologs of retroviral oncogenes (e.g., the ras genes); others are new oncogenes. Tumor suppressor genes or anti-oncogenes have been documented to confer potent negative regulatory controls which are lost due to chromosomal alterations during tumor formation. Functional loss of multiple tumor suppressor genes is believed to be the major event leading to the development of malignancy. Treatment is done till date with the help of radiation therapy, considering the removal of tumor which proceeds with the surgery. Chemotherapy is also used but not to a wide extent and that too has to be done with radiation and research work is still going on the drug discovery for this disease, hence looking towards this fact we opted for the work in this area looking into the successful way of the treatment for oral cancer. The present review is compilation of the data pertaining to biochemistry and molecular biology of oral carcinoma and would certainly provide new insights to explore the development of nutraceuticals based on the hypothesis projected by future studies on computational biotechnology of oral carcinoma.
\end{abstract}

Keywords: Computational Biotechnology of Oral Carcinoma, Neoplasia, Nutraceuticals, Oncogenes, Oral cancer, Papillomavirus.

\section{INTRODUCTION}

Oral cancer or Oral cavity cancer, a subtype of head and neck cancer, is any cancerous tissue growth located in the oral cavity. It may arise as a primary lesion originating in any of the oral tissues, by metastasis from a distant site of origin, or by extension from a neighboring anatomic structure, such as the nasal cavity or the maxillary sinus. Oral cancers may originate in any of the tissues of the mouth, and may be of varied histologic types: teratoma, adenocarcinoma derived from a major or minor salivary gland, lymphoma from tonsillar or other lymphoid tissue, or melanoma from the pigment producing cells of the oral mucosa. Far and away the most common oral cancer is squamous cell carcinoma, originating in the tissues that line the mouth and lips. Oral or mouth cancer most commonly involves the tissue of

*Address correspondence to this author at the School of Biotechnology, IFTM University Lodhipur Rajput, Delhi Road (NH-24), Moradabad 244 001, U.P., India; Tel: +91-591-2360818; Fax: +91-591-2360817;

E-mail: sanjaymishra66@gmail.com the lips or the tongue. It may also occur on the floor of the mouth, cheek lining, gingiva (gums), or palate (roof of the mouth). Most oral cancers look very similar under the microscope and are called squamous cell carcinoma. These are malignant and tend to spread rapidly. Oral cancer incidence ranks fifth in the global cancer burden [1], and a 2- to 3-fold mortality increase has been recorded in eastern and central European countries in the last 3 decades [2]. In India, oral cancer, constituting $9.8 \%$ of an estimated 644,600 incident cancer cases in 1992 [3], ranks first among all cancer cases in males and is the third most common among females in many regions, with age-standardized incidence rates from $7-$ 17/100,000 persons/year [4]; the incidence rate being higher than the western rate of $3-4 / 100,000 /$ year [5].

\section{RISK FACTORS FOR ORAL CANCER}

\section{Tobacco}

Smoking and other tobacco use are associated with about 75 percent of oral cancer cases, caused by irritation of the mucous membranes of the mouth from smoke and heat of 
cigarettes, cigars, and pipes. Tobacco contains over 19 known carcinogens, and the combustion of it, and by products from this process, is the primary mode of involvement. Use of chewing tobacco or snuff causes irritation from direct contact with the mucous membrane.

\section{Alcohol}

Alcohol use is another high-risk factor associated with oral cancer. There is known to be a strong synergistic effect on oral cancer risk when a person is both a heavy smoker and drinker. Their risk is greatly increased compared to a heavy smoker, or a heavy drinker alone. Recent studies, in Australia, Brazil and Germany, point to alcohol-containing mouthwashes as also being etiologic agents in the oral cancer risk family. Constant exposure to these alcohol containing rinses, even in the absence of smoking and drinking, lead to significant increases in the development of oral cancer.

\section{Human Papillomavirus}

Infection with human papillomavirus (HPV), particularly type 16 (there are over 120 types), is a known risk factor and independent causative factor for oral cancer. A fast growing segment of those diagnosed do not present the historic stereotypical demographics. Historically that has been people over 50, blacks over whites (use different terms for race; e.g. African American over Caucasians) 2 to 1, males over females 3 to 1 , and $75 \%$ of the time people who have used tobacco products or are heavy users of alcohol. This new and rapidly growing sub-population between the ages of 20 to 50 years old is predominantly non-smoking, white race individuals, with males slightly outnumbering females. Recent research from Johns Hopkins indicates that HPV is the primary risk factor in this new population of oral cancer patients. HPV16 (along with HPV18) is the same virus responsible for the vast majority of all cervical cancers and is the most common sexually transmitted infection in the US. Oral cancer in this group tends to favor the tonsil and tonsillar pillars, base of the tongue, and the oropharnyx. Recent data suggest that individuals that come to the disease from this particular etiology have a slight survival advantage.

\section{BIOCHEMISTRY AND MOLECULAR BIOLOGY OF ORAL CANCER}

Squamous cell carcinoma of oral cavity is one of the most common malignant neoplasms in India. More than 0.4 million cases are appearing every year. The disfiguring effect associated along with significant mortality make the problem more alarming. Oral cancer is the sixth most common cancer for both sexes in the general population, and the third most common cancer in developing nations [6]. About half of the patients afflicted will die within five years of diagnosis, while surviving patients may be left with severe esthetic and/or functional cooperativity. The International Classification of Diseases defines oral cancer as cancer of the oral cavity and pharynx, including cancer of the lip, tongue, salivary glands, gum, floor and other areas of the mouth, oropharynx, nasopharynx, hypopharynx, pharynx, and other buccal areas. Carcinomas account for $96 \%$ of all oral cancers, $91 \%$ of which are squamous cell carcinomas. The contribution of tobacco and betel quid etc. is already documented as the major cause but recently the role of human papilloma virus (HPV) infection has been suggested by different investigating groups worldwide. Whatever may be the factor(s), reflect the gene(s) probably being associated with cellular growth and differentiation must be affected as cancer in a sense is a genetic disease [7]. Sentence does not make sense.

Oncogenes, gain-of-function mutations of highly regulated normal cellular counterparts (proto-oncogenes), are likely involved in the initiation and progression of oral neoplasia [8]. Cellular oncogenes were initially discovered by the ability of tumor cell DNA to induce transformation in gene transfer assays [9, 10].These experiments have led to the identification of more than 60 cellular oncogenes [11]. Mechanisms of activation of these cellular oncogenes include point mutations and DNA re-arrangements. Several of these cellular oncogenes are homologs of retroviral oncogenes (e.g., the ras genes.

Tumor suppressor genes or anti-oncogenes have been documented to confer potent negative regulatory controls which are lost due to chromosomal alterations during tumor formation. Functional loss of multiple tumor suppressor genes is believed to be the major event leading to the development of malignancy [12, 13]. Unlike oncogenes, which can effect a cellular change through mutation of only one of the two gene copies, tumor suppressor genes are most often inactivated by point mutations, deletions, and rearrangements in both gene copies in a "two-hit" fashion. To date, only three genes - p53, doc-I, and thrombospondin 1-have exhibited tumor suppressor activity in malignant oral keratinocytes.

The genetic polymorphisms of the three enzymes Glutathione-S-transferase, Glutathione peroxdiase, and Glutathione reductase were significantly monitored in reference to oral carcinoma [14]. There was a significant association between cigarette smoking and cancer risk but no significant association between alcohol consumption and cancer risk. The frequency of the GSTM1 null genotype was significantly higher in cancers $(58.7 \%)$ compared with controls $(46.3 \%)$. However, there were no significant differences between controls and patients with oral cavity cancer in the polymorphisms of the GSTT1, GPX and GSR genes. From statistical evaluation on various combinations of genotypes, we did not observe any gene combinations associated with cancer risk. There were also no genetic polymorphisms associated with increased risk of oral cavity cancer among smokers and drinkers [15]. These results revealed that null genotypes of GSTM1 and GSTT1, both individually and in combination, are high penetrance genetic risk factors for developing oral leukoplakia that consequently modulate the risk of developing cancerous oral lesions in habituated $\mathrm{BQ} /$ tobacco chewers of Indian ethnicity, thus our work is in regard of computational information of these protein [15].

Treatment is done with the help of radiation therapy, considering the removal of tumor which proceeds with the surgery. Chemotherapy is also used but not to a wide extent and that too has to be done with radiation and research work is still going on the drug discovery for this disease, hence looking towards this fact we opted for the work in this area looking into the successful way of the treatment for oral cancer [16]. 
Bioinformatics plays an important role in the design of the new drug compounds. The input of bio-computing in the drug discovery is done by providing structural information of the protein targets and their biochemical role in the cell, new therapeutic concepts can developed. It helps in understanding the biological or biochemical mechanism of the disease then often suggests the types of molecules needed for new drugs.

\section{Tobacco-Derived Carcinogens are Involved in the Oral Cancer}

Oral cancer has been causally associated with chewing of tobacco with or without betel quid (BQ) in India and other Asian countries, whereas in western countries, cigarette smoking and heavy alcohol consumption are the main risk factors [17]. Unlike tobacco smoke, which contains many carcinogenic pyrolysis products, such as polycyclic aromatic hydrocarbons (PAH), aldehydes and nitrosamines [18], chewing of tobacco with $\mathrm{BQ}$ increases exposure to carcinogenic tobacco specific nitrosamines. Furthermore, reactive oxygen species (ROS), implicated in multistage carcinogenesis, are also generated in substantial amounts in the oral cavity during chewing $[10,20]$. As an early sign of damage to oral mucosa, $\mathrm{BQ} /$ tobacco chewers often develop Leukoplakia and submucous fibrosis, both well established precancerous lesions, and present an important indicator for oral cancer risk.

\section{GLUTATHIONE S-TRANSFERASE M1 AND T1 NULL GENOTYPES AS RISK FACTORS FOR ORAL LEUKOPLAKIA IN ETHNIC INDIAN BETEL QUID/TOBACCO CHEWERS}

Molecular epidemiological studies have now provided evidence that individual susceptibility to cancer is mediated by both genetic and environmental factors.. The $\mathrm{m}$ (GSTM1) and q (GSTT1) members of the glutathione $S$-transferase (GST) multigene family are candidate cancer-predisposing genes; they are mostly involved in the detoxification of a wide range of environmental and tobacco carcinogens, endogenously produced ROS and lipid peroxidation products, yielding excretable hydrophilic metabolites. The known substrates for GSTM1 include metabolically generated epoxide intermediates of benzopyrene and other PAHs [21], whereas the substrates for GSTT1 include alkyl halides, found in cigarette smoke, and lipid peroxides. The null genotypes that are associated with a lack of enzyme function exist at both these loci and their association with smoking-induced cancer has been investigated. Homozygous deletions of GSTM1 have been associated with higher risk of laryngeal, lung, bladder, colon and gastrointestinal cancers [13-17]. The null genotype of GSTT1 has been reported to be associated with an increased risk of brain [18] and colorectal cancer .The first time a very strong effect of GSTM1 and GSTT1 deletion polymorphisms on the risk of developing oral premalignant lesions and by inference on oral cancer in ethnic Indian $\mathrm{BQ} /$ tobacco chewers.

A highly significantly increased frequency of GSTM1 null genotype in all leukoplakia cases of $81.6 \%$, compared with $17 \%$ in controls. Individuals with GSTMI had a 22 -fold higher risk of leukoplakia [22]. The homozygous null genotype GSTT1 was found in $75.5 \%$ of cases and in $22 \%$ of con- trols, conferring an 11-fold higher risk. A total of $60.2 \%$ of the cases presented homozygous deletion of both GSTT1 and GSTM1, but none was detected among healthy control chewers, revealing a potentiation of risk in subjects with both null genotypes for oral cancer when they chew tobacco. The combined null genotype of both GSTM1 and GSTT1 conferred a highly significant risk for oral leukoplakia_when compared with the category of at least copy of the genes present [23]. Thus, the GSTM1 and GSTT1 deletion genotype alone and even more in combination was demonstrated to highly predispose habitual $\mathrm{BQ}$ chewers to the development of oral leukoplakia, a precancerous lesion.

The GSTM1gene has been mapped to chromosome 1p13.3 and GSTT1 gene to chromosome 22q11.2. As loss of heterozygosity $(\mathrm{LOH})$ has also been reported in oral cancers on chromosomes 1p11-13 and chromosome 22q13; gene deletion due to $\mathrm{LOH}$ on the specific amplicons can at present not be excluded as an additional contributory factor. Studies are underway to verify this possibility [24]. It is suggested that carcinogenic intermediates derived from $\mathrm{BQ} /$ tobacco or generated during habitual chewing are substrates for GSTT1 and GSTM1 enzymes and that oral leukoplakia risk is increased more than additively for individuals who have both GSTT1 and GSTM1 null genotypes [24].

\section{GST Null Genotype and Antioxidants: Risk for Oral Cancer}

The study was undertaken to detect the gene polymorphism of detoxification enzymes and estimate the antioxidant enzyme status in patients with oral cancer, oral leukoplakia and oral submucous fibrosis (OSF). The GSTM1 and GSTT1 gene polymorphism was evaluated using polymerase chain reaction; the antioxidant enzyme was estimated using biochemical methods. Statistical analyses were performed using student t-test and odds-ratio to estimate relative risk (RR). The RR at 95\% confidence interval (CI) for GSTM1 and GSTT1 was statistically significant for all groups. The mean values of glutathione were significantly raised in all groups [25]. The mean values of ceruloplasmin and malonaldehyde was statistically significant among cancer and OSF patients but was insignificant in smokers and cases with leukoplakia. Which made to conclude that, Several genes perform the same function which implies the need to test for several genetic polymorphisms to identify individuals at high risk. The level of antioxidant enzymes correlate with the degree of oxidative damage [26].

Investigation was made those genetic polymorphisms in GSTM1\& GSTT1 and the association with the risk of oral cancer in the Jakarta population. A total of 81 cases and 162 controls matched for age and sex were selected from 5 hospitals in Jakarta. Socio demographic data using questionnaires were obtained and peripheral blood samples were collected with informed consent for PCR-RFLP assay [27]. Conditional logistic regression analysis was performed to obtain the association between the risk of oral cancer and GSTM1\& GSTT1 polymorphisms. GSTM1 and GSTT1 null were slightly over represented among cases $(60.5 \%$ and $45.7 \%$ respectively) compared to controls $(55.6 \%$ and $41.4 \%$ respectively), but no statistically significant differences were observed. The odds ratio of null GSTM1 and GSTT1 genotypes was slightly higher compared to wild type genotypes [27]. 


\section{Data of Different Countries}

With further studies we also came to the fact that GSTM1 null genotype significantly increases susceptibility to oral cancer in Asians. Ethnic differences in the prevalence of the GSTM1 null genotypes have been reported to vary between $22-35 \%$ in Africans, $38-67 \%$ in Caucasians and $33-63 \%$ in East Asian populations [10, 28]. The Pacific islanders (Oceania) have the highest reported frequency, GSTM1 *2 ranges from $64 \%$ to as high as $100 \%$ in Kiribati natives [29]. The GSTT1 $* 2$ genotype varies from $10-18 \%$ in Caucasians [21- 23] to 58\% in the Chinese [24]. To our knowledge, this is the first report on the prevalence of these genotypes in an ethnic Indian population and among premalignant leukoplakia cases from India.

\section{THE STUDY OF THE PROTEINS AND LIGANDS OF INTEREST}

\section{Glutathione s-Transferase}

Enzymes of the Glutathione $-\boldsymbol{S}$-transferase (GST) family are composed of many cytosolic, mitochondrial, and microsomal (now designated as MAPEG) proteins. GSTs are present in eukaryotes and in prokaryotes, where they catalyze a variety of reactions and accept endogenous and xenobiotic substrates [30].

GSTs can constitute up to $10 \%$ of cytosolic protein in some mammalian organs. GSTs catalyse the conjugation of reduced glutathione - via a sulfhydryl group - to electrophilic centers on a wide variety of substrates [31]. This activity detoxifies endogenous compounds such as peroxidised lipids, as well as breakdown of xenobiotics. GSTs may also bind toxins and function as transport proteins, and, therefore, an early term for GSTs was "ligandin". The mammalian GST super-family consists of cytosolic dimeric isoenzymes of 45-55 kDa size that have been assigned to at least four classes: Alpha, $\mathrm{Mu}, \mathrm{Pi}$ and Theta (Table 1) [31].

Table 1. The Following is a List of Human Glutathione Stransferases

\begin{tabular}{|c|c|}
\hline Class & Members \\
\hline Alpha & GSTA1, GSTA2, GSTA3 3 GSTA4, GSTA5 \\
\hline kappa & $\underline{\text { GSTK1 }}$ \\
\hline $\mathrm{Mu}$ & GSTM1, GSTM1L, GSTM2, GSTM3, GSTM4, GSTM5 \\
\hline omega & GSTO1, GSTO2 \\
\hline Pi & $\underline{\text { GSTP1 }}$ \\
\hline Theta & GSTT1, GSTT2 \\
\hline microsomal & $\underline{\text { MGST1}}, \underline{\text { MGST2}}, \underline{\text { MGST3 }}$ \\
\hline
\end{tabular}

Most mammalian isoenzymes have affinity for the substrate 1-chloro-2,4-dinitrobenzene (CDNB), and spectrophotometric assays utilizing this substrate are commonly used to report GST activity. However, some endogenous compounds, e.g., bilirubin, can inhibit the activity of GSTs. In mammals, GST isoforms have cell specific distributions (e.g., alpha GST in hepatocytes and pi GST in the biliary tract of the human liver) [32, 33].

A family of enzymes that utilizes glutathione in reactions contributing to the transformation of a wide range of compounds, including carcinogens, therapeutic drugs, and products of oxidative stress [32]. These enzymes play a key role in the detoxification of such substances. The glutathione S-transferases act by catalyzing the reaction of glutathione with an acceptor molecule to form an S-substituted glutathione ( $\mathrm{S}=$ sulfur). See also: Glutathione $\mathrm{S}$-transferase omega-1 [33].

\section{GST-tags and the GST Pull-Down Assay}

Genetic engineers have used glutathione S-transferase to create the GST gene fusion system. This system is used to purify and detect proteins of interest. In a GST gene fusion system, the GST sequence is incorporated into an expression vector alongside the gene sequence encoding the protein of interest. Induction of protein expression from the vector's promoter results in expression of a fusion protein: the protein of interest fused to the GST protein. This GST-fusion protein can then be purified from cells via its high affinity for glutathione [34].

The fusion proteins offer an important biological assay for direct protein-to-protein interactions. For instance, to demonstrate that caveolin (a membrane protein) binds to eNOS (a catalytic protein) a GST-caveolin fusion protein would be generated. Assay beads, coated with the tripeptide glutathione, strongly bind the GST fusion protein (GSTcaveolin, in this example). It is noted that, if caveolin binds eNOS, then GST-caveolin will also bind eNOS, and this eNOS will therefore be present on assay beads [35]. GST is commonly used to create fusion proteins. The tag has the size of 220 amino acids (roughly $26 \mathrm{KDa}$ ), which, compared to other tags like the myc- or the FLAG-tag, is quite big. It is fused to the N-terminus of a protein. However, many commercially-available sources of GST-tagged plasmids include a thrombin domain for cleavage of the GST tag during protein purification [35].

A GST-tag is often used to separate and purify proteins that contain the GST-fusion. GST-fusion proteins can be produced in Escherichia coli, as recombinant proteins. The GST part binds its substrate, glutathione. Agarose beads can be coated with glutathione, and such glutathione-Agarose beads bind GST-proteins. These beads are then washed, to remove contaminating bacterial proteins. Adding free glutathione to beads that bind purified GST-proteins will release the GST-protein in solution [36].

\section{Glutathione Peroxidase}

Glutathione peroxidase is the general name of an enzyme family with peroxidase activity whose main biological role is to protect the organism from oxidative damage [37]. The biochemical function of glutathione peroxidase is to reduce lipid hydroperoxides to their corresponding alcohols and to reduce free hydrogen peroxide to water.

There are several isozymes encoded by different genes, which vary in celullar location and substrate specificity. Glutathione peroxidase 1 (GPx1) is the most abundant version, found in the cytoplasm of nearly all mammalian tissues, 
whose preferred substrate is hydrogen peroxide. Glutathione peroxidase 4 (GPx4) has a high preference for lipid hydroperoxides; it is expressed in nearly every mammalian cell, though at much lower levels. Glutathione peroxidase 2 is an intestinal and extracellular enzyme, while glutathione peroxidase 3 is extracellular, especially aboundant in plasma [38]. So far, eight different isoforms of glutathione peroxidase (GPx 1-8) have been identified in humans. An example reaction that glutathione peroxidase catalyzes is:

$$
2 \mathrm{GSH}+\mathrm{H}_{2} \mathrm{O}_{2} \rightarrow \mathrm{GS}-\mathrm{SG}+2 \mathrm{H}_{2} \mathrm{O},
$$

where GSH represents reduced monomeric glutathione, and GS-SG represents glutathione disulfide.

Mammalian GPx1, GPx2, GPx3, and GPx4 have been shown to be selenium-containing enzymes, whereas GPx6 is a selenoprotein in humans with cysteine - containing homologues in rodents. GPx1, GPx2, and GPx3 are homotetrameric proteins, whereas GPx4 has a monomeric structure. As the integrity of the cellular and subcellular membranes depends heavily on glutathione peroxidase, the antioxidative protective system of glutathione peroxidase itself depends heavily on the presence of selenium.

The mechanism is at the Selenocystein site, which is in a Se (-) form as resting state. This is oxidized by the peroxide to $\mathrm{SeOH}$ which is then trapped by a GSH molecule to Se-SG and by another GSH molecule to $\mathrm{Se}(-)$ again, releasing a GSSG by-product.

\section{Glutathione Reductase}

Glutathione reductase, also known as GSR or GR, is an enzyme that reduces glutathione disulfide (GSSG) to the sulfhydryl form GSH, which is an important cellular antioxidant $[39,40]$. For every mole of oxidized glutathione (GSSG), one mole of NADPH is required to reduce GSSG to GSH. The enzyme forms a FAD bound homodimer. The glutathione reductase is conserved between all kingdoms. In bacteria, yeasts, and animals, one glutathione reductase gene is found; however, in plant genomes, two GR genes are encoded. Drosophila and Trypanosomes do not have any GR at all [40]. In these organisms, glutathione reduction is performed by either the thioredoxin or the trypanothione system, respectively.

NADPH reduces FAD present in GSR to produce a transient $\mathrm{FADH}^{-}$anion. This anion then quickly breaks a disulfide bond $\left(\mathrm{Cys}_{58}-\mathrm{Cys}_{63}\right)$ and leads to $\mathrm{Cys}_{63}$ 's nucleophilically attacking the nearest sulfide unit in the GSSG molecule (promoted by $\mathrm{His}_{467}$ ), which creates a mixed disulfide bond $\left(\mathrm{GS}^{-} \mathrm{Cys}_{58}\right)$ and a $\mathrm{GS}^{-}$anion. His ${ }_{467}$ of GSR then protonates the $\mathrm{GS}^{-}$anion to form the first GSH. Next, Cys 63 nucleophilically attacks the sulfide of $\mathrm{Cys}_{58}$, releasing a $\mathrm{GS}^{-}$anion, which, in turn, picks up a solvent proton and is released from the enzyme, thereby creating the second GSH. So, for every GSSG and NADPH, two reduced GSH molecules are gained, which can again act as antioxidants scavenging reactive oxygen species in the cell $[32,41]$. In cells exposed to high levels of oxidative stress, like red blood cells, up to $10 \%$ of the glucose consumption may be directed to the pentose phosphate pathway (PPP) for production of the NADPH needed for this reaction. In the case of erythrocytes, if the PPP is non-functional, then the oxidative stress in the cell will lead to cell lysis and anemia. The activity of glutathione reductase is used as indicator for oxidative stress [42]. The activity can be monitored by the NADPH consumption, with absorbance at $340 \mathrm{~nm}$, or the formed GSH can be visualized by Ellman's reagent [43]. Alternatively the activity can be measured using roGFP (redox-sensitive Green Fluorescent Protein) [44].

\section{LIGANDS OF SIGNIFICANCE}

\section{Iodoacetamide}

2-Iodoacetamide is an alkylating sulfhydryl reagent used for peptide mapping purposes. Its actions are similar to those of iodoacetate. It is commonly used to bind covalently with cysteine, so the protein cannot form disulfide bond [44]. After that reducing agents are added to react with any excess alkylating agent. Also used in ubiquitin studies as an inhibitor of deubiquitinase enzymes (DUBs) because it alkylates the cysteine residues at the DUB active site. In laboratory mice it has been shown to cause skin tumors and negatively affect reproductive ability. It may act as a human carcinogen, and may cause reproductive damage. As far as properties computed from structure are concerned, are shown in Table 2 .

Table 2. Properties Computed from Structure: http:// pubchem.ncbi.nlm.nih.gov/summary/summary.cgi? cid $=3727 \&$ loc $=$ ec_res - top

\begin{tabular}{|c|c|}
\hline Molecular Weight & $184.96373[\mathrm{~g} / \mathrm{mol}]$ \\
\hline Molecular Formula & $\mathrm{C}_{2} \mathrm{H}_{4} \mathrm{INO}$ \\
\hline $\mathrm{X} \log \mathrm{P} 3$ & -0.2 \\
\hline H-Bond Donor & 1 \\
\hline H-Bond Acceptor & 1 \\
\hline Rotatable Bond Count & 1 \\
\hline Tautomer Count & 2 \\
\hline Exact Mass & 184.933757 \\
\hline MonoIsotopic Mass & 184.933757 \\
\hline Topological Polar Surface Area & 43.1 \\
\hline Heavy Atom Count & 5 \\
\hline Formal Charge & 0 \\
\hline Complexity & 44.9 \\
\hline Isotope Atom Count & 0 \\
\hline Defined Atom StereoCenter Count & 0 \\
\hline Undefined Atom StereoCenter Count & 0 \\
\hline Defined Bond StereoCenter Count & 0 \\
\hline Undefined Bond StereoCenter Count & 0 \\
\hline Covalently-Bonded Unit Count & 1 \\
\hline
\end{tabular}

IUPAC Name: 2-iodoacetamide Canonical SMILES: $\mathrm{C}(\mathrm{C}(=\mathrm{O}) \mathrm{N}) \mathrm{I}$ 


\section{Beta Mercaptoethanol}

2-Mercaptoethanol (also $\beta$-mercaptoethanol, BME, $2 \mathrm{BME}$ or $\beta$-met) is the chemical compound with the formula $\mathrm{HOCH}_{2} \mathrm{CH}_{2} \mathrm{SH}$. It is a hybrid of ethylene glycol, $\mathrm{HOCH}_{2} \mathrm{CH}_{2} \mathrm{OH}$, and 1,2-ethanedithiol, $\mathrm{HSCH}_{2} \mathrm{CH}_{2} \mathrm{SH}$. ME or $\beta \mathrm{ME}$, as it is commonly abbreviated, is used to reduce disulfide bonds and can act as a biological antioxidant by scavenging hydroxyl radicals (amongst others) [45]. It is widely used because the hydroxyl group confers solubility in water and lowers the volatility. Due to its diminished vapor pressure, its odour, while unpleasant, is less objectionable than related thiols. ME may be prepared by the action of hydrogen sulfide on ethylene oxide:

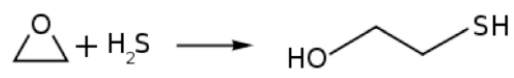

Some proteins can be denatured by 2-mercaptoethanol via its ability to cleave disulfide bonds:

cysS-Scys $+2 \mathrm{HOCH}_{2} \mathrm{CH}_{2} \mathrm{SH} \rightarrow 2 \mathrm{cysSH}+\mathrm{HOCH}_{2} \mathrm{CH}_{2}$ $\mathrm{S}-\mathrm{SCH}_{2} \mathrm{CH}_{2} \mathrm{OH}$

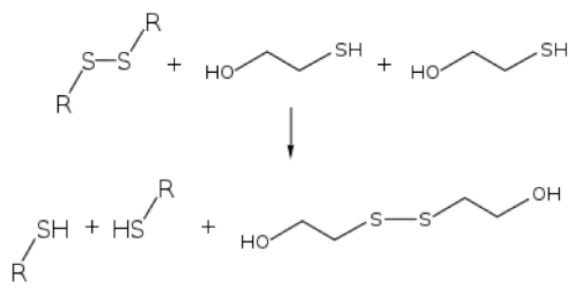

By breaking the S-S bonds, both the tertiary structure and the quaternary structure of some proteins can be disrupted [45]. Because of its ability to disrupt the structure of proteins, it is used in the analysis of proteins, for instance, to ensure that a protein solution contains monomeric protein molecules, instead of disulfide linked dimers or higher order oligomers. 2-Mercaptoethanol is often used interchangeably with dithiothreitol (DTT) or the odorless tris (2carboxyethyl) phosphine (TCEP) in biological applications. 2-Mercaptoethanol is used in some RNA isolation procedures to eliminate ribonuclease released during cell lysis. Numerous disulfide bonds make ribonucleases very stable enzymes, so 2-mercaptoethanol is used to reduce these disulfide bonds and irreversibly denature the proteins. This prevents them from digesting the RNA during its extraction procedure [45, 46]. 2-Mercaptoethanol is considered a toxin, causing irritation to the nasal passageways and respiratory tract upon inhalation, irritation to the skin, vomiting and stomach pain through ingestion, and potentially death if severe exposure occurs [46]. As far as properties computed from structure are concerned, are shown in Table 3.

\section{DTT}

Dithiothreitol (DTT) is the common name for a smallmolecule redox reagent known as Cleland's reagent [47]. DTT's formula is $\mathrm{C}_{4} \mathrm{H}_{10} \mathrm{O}_{2} \mathrm{~S}_{2}$ and the molecular structure of its reduced form is shown at the right; its oxidized form is a disulfide-bonded 6-membered ring (shown below). Its name derives from the four-carbon sugar, threose. DTT has an epimeric ('sister') compound, dithioerythritol (DTE) [46, 47].
Table 3. Properties Computed from Structure: http:// pubchem.ncbi.nIm.nih.gov/summary/summary.cgi? cid $=1567 \&$ loc $=$ ec_res - top

\begin{tabular}{|l|l|}
\hline Molecular Weight & $\mathbf{7 8 . 1 3 3 4 4}$ [g/mol] \\
\hline \hline Molecular Formula & $\mathrm{C}_{2} \mathrm{H}_{6} \mathrm{OS}$ \\
XLogP3-AA & -0.2 \\
\hline H-Bond Donor & 1 \\
\hline H-Bond Acceptor & 1 \\
\hline Rotatable Bond Count & 1 \\
\hline Exact Mass & 78.013936 \\
\hline MonoIsotopic Mass & 78.013936 \\
\hline Topological Polar Surface Area & 20.2 \\
\hline Heavy Atom Count & 4 \\
\hline Formal Charge & 0 \\
\hline Complexity & 10 \\
\hline Isotope Atom Count & 0 \\
\hline Defined Atom StereoCenter Count & 0 \\
\hline Undefined Atom StereoCenter Count & 0 \\
\hline Defined Bond StereoCenter Count & 0 \\
\hline Undefined Bond StereoCenter Count & 0 \\
\hline Covalently-Bonded Unit Count & 1 \\
\hline
\end{tabular}

IUPAC Name: 2-sulfanylethanol

Canonical SMILES: C(CS)O

DTT is an unusually strong reducing agent, owing to its high conformational propensity to form a six-membered ring with an internal disulfide bond. It has a redox potential of $-0.33 \mathrm{~V}$ at $\mathrm{pH}$ 7. The reduction of a typical disulfide bond proceeds by two sequential thiol-disulfide exchange reactions and is illustrated below. The intermediate mixed-disulfide state is unstable (i.e., poorly populated) because the second thiol of DTT has a high propensity to close the ring, forming oxidized DTT and leaving behind a reduced disulfide bond. The reducing power of DTT is limited to $\mathrm{pH}$ values above $\sim 7$, since only the negatively charged thiolate form $-\mathrm{S}^{-}$is reactive (the protonated thiol form - $\mathrm{SH}$ is not); the $\mathrm{pKa}$ of the thiol groups is 9.2 and 10.1. A common use of DTT is as a reducing or "deprotecting" agent for thiolated DNA. The terminal sulfur atoms of thiolated DNA have a tendency to form dimers in solution, especially in the presence of oxygen. Dimerization greatly lowers the efficiency of subsequent coupling reactions such as DNA immobilization on gold in biosensors [47]. Typically DTT is mixed with a DNA solution and allowed to react, and then is removed by filtration (for the solid catalyst) or by chromatography (for the liquid form). The DTT removal procedure is often called "desalting." 
DTT is frequently used to reduce the disulfide bonds of proteins and, more generally, to prevent intramolecular and intermolecular disulfide bonds from forming between cysteine residues of proteins. However, even DTT cannot reduce buried (solvent-inaccessible) disulfide bonds, so reduction of disulfide bonds is sometimes carried out under denaturing conditions (e.g., at high temperatures, or in the presence of a strong denaturant such as $6 \mathrm{M}$ guanidinium hydrochloride, $8 \mathrm{M}$ urea, or $1 \%$ sodium dodecylsulfate). Conversely, the solvent exposure of different disulfide bonds can be assayed by their rate of reduction in the presence of DTT. DTT can also be used as an oxidizing agent. Its principal advantage is that effectively no mixed-disulfide species are populated, in contrast to other agents such as glutathione. In very rare cases, a DTT adduct may be formed, i.e., the two sulfur atoms of DTT may form disulfide bonds to different sulfur atoms; in such cases, DTT cannot cyclize since it has no remaining free thiols. Due to air oxidation, DTT is a relatively unstable compound whose useful life can be extended by refrigeration and handling in an inert atmosphere. Since protonated sulfurs have lowered nucleophilicities, DTT becomes less potent as the $\mathrm{pH}$ lowers. Tris (2-carboxyethyl) phosphine $\mathrm{HCl}$ (TCEP hydrochloride) is an alternative which is more stable and works even at low $\mathrm{pH}[47,48]$. As far as properties computed from structure are concerned, are shown in Table 4.

Table 4. Properties Computed from Structure

\begin{tabular}{|l|l|}
\hline Molecular Weight & $\mathbf{1 5 4 . 2 5 1}$ [g/mol] \\
\hline \hline Molecular Formula & $\mathrm{C}_{4} \mathrm{H}_{10} \mathrm{O}_{2} \mathrm{~S}_{2}$ \\
\hline XLogP3-AA & -0.4 \\
\hline H-Bond Donor & 2 \\
\hline H-Bond Acceptor & 2 \\
\hline Rotatable Bond Count & 3 \\
\hline Exact Mass & 154.012221 \\
\hline MonoIsotopic Mass & 154.012221 \\
\hline Topological Polar Surface Area & 40.5 \\
\hline Heavy Atom Count & 8 \\
\hline Formal Charge & 0 \\
\hline Complexity & 0 \\
\hline Isotope Atom Count & 0 \\
\hline Defined Atom StereoCenter Count & 0 \\
\hline Undefined Atom StereoCenter Count & 0 \\
\hline
\end{tabular}

IUPAC Name: 1,4-bis(sulfanyl)butane-2,3-diol

Canonical SMILES: C(C(C(CS)O)O $) \mathrm{S}$
GLUTATHIONE -S- TRANSFERESE M1

\section{FASTA Format:}

>gi|49456691|emb|CAG46666.1| GSTM1 [Homo sapiens] MPMILGYWDIRGLAHAIRLLLEYTDSSYEEKKYTMG DAPDYDRSQWLNEKFKLGLDFPNLPYLIDGAHKITQSNAILCYIARKHNLCGETEEEKIRVDILENQTMDNHMQLGMICYNPEFEKLKPKYLEELPEKLKLYSEFLGKRPWFAGNKITFVDFLVYDVLDLHRIFEPNCLDAFPNLKDFISRFEGLEKISAYMKSSRFLPRPVFSKMAVWGNK

\section{GLUTATHIONE PEROXIDASE}

\section{FASTA Format:}

>gi|577062|emb|CAA31992.1| glutathione peroxidase [Homo sapiens]

MCAARLAAAAAQSVYAFSARPLAGGEPVSLGSLRGKVLLIENVASLXGTTVRDYTQMNELQRRLGPRGLVVLGFPCNQFGHQENAKNEEIQNSLKYVRPGGGFEPNFMLFEKCEVNGAGAHPLFAFLREALPAPSDDATALMTDPKLITWSPVCRNDVAWNFEKFLVGPDGVPLRRYSRRFQTIDIEPDIEALLSQGPSCA

\section{GLUTATHIONE REDUCTASE}

\section{FASTA Format:}

$>$ gi|31829|emb|CAA38367.1| glutathione reductase [Homo sapiens]

EFMHDHADYGFPSCEGKFNRVIKEKRDAYVSRLNAIYQNLTKSHIEIIRGHAAFTSDKPTIEVSGKKYTAPHILIAGGMPSTPHESQIPGASLGISDGFFQLEELPGRSVIVGAYIAVEMAGILSALGSKTSLIRHDKVLRSFDSMISTNCTELENAGVEVLKFSQVKEVKTLSGLEVSMVTAVPGRLPVTMIPDVDCLLWAIGRVPNTDLSLNKLGIQTDDKGHIIVEF

\section{CONCLUDING REMARKS}

Conclusively, the present review aims to compile and update the informations available pertaining to the biochemical and molecular mechanisms of oral carcinogenesis and explore the future perspectives where the field of Cancer Biology is reflecting ventures. The prevalence and mortality due to one of the multifactorial polygenic diseases, namely oral cancer vary depending upon genetic susceptibility and environmental precursors because they have identifiable mendelian subsets [10]. Rapid changes in diet and lifestyle, may influence heritability of the variant phenotypes that are dependent on the nutraceutical or functional food supplementation for their expression. It is possible to recognize the interaction of specific nutraceuticals, with the genetic code possessed by all nucleated cells. There is evidence that South Asians have an increased susceptibility to oral cancer even at younger age, which may be due to interaction of gene and nutraceutical environment. Oncologists have straightforwardly followed the old saying that: necessity is the principal mother of an invention [40, 47-49]. The desire to be more precise and comprehensive in their studies has led to the invention of some of the most innovative techniques such as proteomics, comparative genomic hybridization, microar- 
rays, nanobiotechnology etc. A number of Biotech companies and Cancer laboratories are on the way to hunt for anticancer drugs and biochemical/molecular markers for diagnose and check cancers. These innovative approaches and the modern oncologists have made the efforts in this specific field with exciting results and have provided the hope, which ultimately leads towards the combat against carcinoma would be winning over. Finally, the compilation of the literature embodied in this review provides new insights into further exploring to identify suitable biochemical and/or molecular markers for the early detection of pre-neoplastic lesions, as well as novel targets for its pharmacological intervention employing bioinformatics based modern biotechnological tools.

\section{CONFLICT OF INTEREST}

None declared.

\section{ACKNOWLEDGEMENTS}

The present paper is a piece of $\mathrm{Ph} . \mathrm{D}$. studies carried out by author, RS registered at Integral University, Lucknow, UP, India.

\section{REFERENCES}

[1] Parkin DM, Pisani P, Ferlay J. Estimates of the worldwide incidence of eighteen major cancers in 1985. Int J Cancer 1993; 54: 594-606.

[2] Coleman MP, Esteve J, Damiecki P, Arslan A, Renard H. Trends in Cancer Incidence and Mortality. IARC Scientific Publications No. 121, IARC, Lyon, 1993.

[3] Indian Council of Medical Research (19922) National Cancer Registry Programme: Biennial Report 1988-1989. ICMR, New Delhi, India.

[4] Parkin DM, Whelan SL, Ferlay J, Raymond LA, Young J. Cancer Incidence in Five Continents Vol. VII. IARC Scientific Publications No. 143, IARC, Lyon, 1997.

[5] Macfarlane GJ, Boyle P, Evstifeeva TV, Robertson C, Scully C. (1994) Rising trends of oral cancer mortality among males worldwide: the return of an old public health problem. Cancer Causes Control 1994; 5(3): 259-65.

[6] La Vecchia C, Tavani A, Franceschi S, Levi F, Corrao G, Negri E. Epidemiology and prevention of oral cancer. Oral Oncol 1997; 33: 302-12.

[7] IARC Tobacco smoking. In IARC Monographs on the Evaluation of the Carcinogenic Risk of Chemicals to Humans, Vol. 38. IARC Scientific Publications, Lyon, 1986.

[8] Hoffmann D, Hecht SS. (1985) Nicotine-derived $N$-nitrosamines and tobacco-related cancer: current status and future directions. Cancer Res 1985; 45: 935-44.

[9] Nair UJ, Nair J, Friesen MD, Bartsch H, Ohshima H. Orthoand meta-tyrosine formation from phenylalanine in human saliva as a marker of hydroxyl radical generation during betel quid chewing. Carcinogenesis 1995; 16: 1195-8.

[10] Mishra S, Singh RB, Dwivedi SP, et al. Effect of nutraceuticals on genetic expressions. Open Nutra J 2009; 2: 70-80.

[11] Nair UJ, Obe G, Friesen M, Goldberg MT, Bartsch H. Role of lime in the generation of reactive oxygen species from betel-quid ingredients. Environ Health Perspect 1992; 98: 203-5.

[12] Hayes JD, Pulford DJ. The glutathione $S$-transferase supergene family: regulation of GST and the contribution of the isoenzymes to cancer chemoprotection and drug resistance. Crit Rev Biochem Mol Biol 1995; 30: 445-600.

[13] Brockmo"ller J, Kerb R, Drakoulis N, Staffeldt B, Roots I. (1994) Glutathione $S$-transferase M1 and its variants A and B as host factors of bladder cancer susceptibility: a case-control study. Cancer Res 1994; 54: 4103-11.

[14] Chenevix TG, Young J, Coggan M, Board P. Glutathione $S$ transferase M1 and T1 polymorphisms: susceptibility to colon cancer and age of onset. Carcinogenesis 1995; 16: 1655-7.
[15] Hayashi S, Watanabe J, Kawajiri K. High susceptibility to lung cancer analyzed in terms of combined genotypes of P4501A1 and Muclass glutathione $S$-transferase genes. Japn J Cancer Res 1992; 83: 866-70.

[16] Katoh T, Nagata N, Kuroda Y, et al. Glutathione $S$-transferase M1 (GSTM1) and T1 (GSTT1) genetic polymorphism and susceptibility to gastric and colorectal adenocarcinoma. Carcinogenesis 1996; 17: 1855-9.

[17] Lafuente A, Pujol F, Carretero P, Villa JP, Cuchi A. Human Glutathione $S$-transferase mu (GST mu) deficiency as a marker for the susceptibility to bladder and larynx cancer among smokers. Cancer Lett 1993; 68: 49-54.

[18] Elexpuru CJ, Buxton N, Kandula V, et al. Susceptibility to astrocytoma and meningioma: influence of allelism at glutathione Stransferase(GSTT1 and GSTM1) and cytochrome P-450 (CYP2D6) loci. Cancer Res 1995; 55: 4237-9.

[19] Rebbeck TR. Molecular epidemiology of the human glutathione $S$ transferase genotypes GSTM1 and GSTT1 in cancer susceptibility. Cancer Epidemiol Biomarkers Prev 1997; 6: 733-43.

[20] Board P, Coggan M, Johnston P, Ross V, Suzuki T, Webb G. Genetic heterogeneity of the human glutathione transferases: a complex of gene families. Pharmacol Ther 1990; 48: 357-69.

[21] Chenevix TG, Young J, Coggan M, Board P. Glutathione $S$ transferase M1 and T1 polymorphisms: susceptibility to colon cancer and age of onset. Carcinogenesis 1995; 16: 1655-7.

[22] Elexpuru CJ, Buxton N, Kandula V, et al. Susceptibility to astrocytoma and meningioma: influence of allelism at glutathione Stransferase (GSTT1 and GSTM1) and cytochrome P-450 (CYP2D6) loci. Cancer Res 1995; 55: 4237-9.

[23] Nelson HH, Wiencke JK, Christiani DC, et al. Ethnic differences in the prevalence of the homozygous deleted genotype of glutathione $S$-transferase theta. Carcinogenesis 1995; 16: 1243-5.

[24] Lee EJ, Wong JY, Yeoh PN, Gong NH. Glutathione Stransferasetheta (GSTT1) genetic polymorphism among Chinese, Malays and Indians in Singapore. Pharmacogenetics 1995; 5: 332-4.

[25] Muller FL, Lustgarten MS, Jang Y, Richardson A, Van Remmen H. Trends in oxidative aging theories. Free Radic Biol Med 2007; 43(4): 477-503.

[26] Meister A. Glutathione metabolism and its selective modification. J Biol Chem 1988; 263(33): 17205-8.

[27] Mannervik B. The enzymes of glutathione metabolism: an overview. Biochem Soc Trans 1987; 15(4): 717-8.

[28] Kanzok SM, Fechner A, Bauer H, et al. Substitution of the thioredoxin system for glutathione reductase in Drosophila melanogaster. Science 2001; 291(5504): 643-6.

[29] Champe PC, Harvey RA, Ferrier DR. Biochemistry, Fourth Edition. Lippincott Williams and Wilkins, 2008.

[30] Marty L, Siala W, Schwarzländer M, et al. The NADPH-dependent thioredoxin system constitutes a functional backup for cytosolic glutathione reductase in Arabidopsis. Proc Natl Acad Sci U S A 2009; 106(22): 9109-14.

[31] Smythe CV. The reactions of Iodoacetate and of Iodoacetamide with various Sulfhydryl groups, with Urease, and with Yeast preparations. J Biol Chem 1936; 114: 601-12.

[32] Anson ML. The reactions of Iodine and Iodoacetamide with native Egg Albumin. J Gen Physiol 1940; 23: 321-31.

[33] Sharma V, Kalim S, Srivastava MK, Nanda S, Mishra S. Oxidative stress and coxsackievirus infections act as mediators of beta cell damage: a review. SRE 2009; 4 (2): 42-58.

[34] Knight JJ. 2-Mercaptoethanol in Encyclopedia of Reagents for Organic Synthesis (Ed: L. Paquette) 2004, J. Wiley \& Sons, New York.

[35] 2-Mercaptoethanol. Chemicalland21.com. http://www.chemicalland21. $\mathrm{com} /$ specialtychem/finechem/2-MERCAPTOETHANOL.htm.

[36] Nelson DR, Lehninger AL, Cox M. Lehninger principles of biochemistry. New York: W.H. Freeman. pp. 148, 2005.

[37] Material Safety Data Sheet. JT Baker. http://www.jtbaker.com/ msds/englishhtml/m1209.htm.

[38] Combet C, Jambon M, Deléage G, Geourjon C. Institut de Biologie et Chimie des Protéines, IBCP-CNRS UMR 5086, Pôle Bioinformatique Lyonnais, Bioinformatics and structural NMR, 7, Passage du Vercors, 69367 Lyon Cedex 07, France.

[39] Morris GM, Goodsell DS, Halliday RS, et al. Automated docking using a Lamarckian genetic algorithm and an empirical binding free energy function journal. J Comput Chem 1998; 19: $1639-62$. 
[40] Csizmadia F. JChem: Java applets and modules supporting chemical database handling from web browsers. J Chem Inf Comp Sci 2000; 40: $323-4$.

[41] Nagpal JK, Das BR. Oral cancer: reviewing the present understanding of its molecular mechanism and exploring the future directions for its effective management. Oral Oncol 2003; 39(3): 213-21.

[42] Nanda S, Mishra S, Varshney VP, Singh RB. A biotechnological approach to apoptosis of somatic and germ cells in living organisms. Open Nutra J 2010; 3: 81-93.

[43] Mishra S, Tyagi A, Dwivedi, SP. Invited Review: Regulation of programmed cell death or apoptosis in living organisms: A biotechnological approach. Biotechnol Bioinf Bioeng 2010; 1: 1-19.

[44] Nanda S, Varshney VP, Chauhan N, Srivastava R, Singh RB, Mishra S. Synergistic cytotoxic effect of Syzygium aromaticum extract and gemicitabine on human cervical cancer cell line. J Advance Res Biological Sci 2009; 1(2): 172-81.
[45] López-Alonso JP, Bruix M, Font J, et al. NMR spectroscopy reveals that RNase A is chiefly denatured in $40 \%$ acetic acid: implications for oligomer formation by $3 \mathrm{D}$ domain swapping. J Am Chem Soc 2010; 132: 1621-30.

[46] Nelson DR, Lehninger AL, Cox M. Lehninger Principles of Biochemistry. New York: W.H. Freeman. pp. 148, 2005.

[47] Ruegg UT, Rudinger J. Cleavage of disulfide bonds in proteins. Methods Enzymol 1997; 47: 111.

[48] Duchardt F, Ruttekolk IR, Verdurmen WP, et al. A cell-penetrating peptide derived from human lactoferrin with conformationdependent uptake efficiency. J Biol Chem 2009; 284(52): 36099108.

[49] Mishra S, Dwivedi SP, Dwivedi, Singh RB. Immune response and possible causes of CD4+T-cell depletion in human immunodeficiency virus (HIV) - 1 infection. Open Nutra J 2009; 2: 46-51.

(C) Srivastava et al.; Licensee Bentham Open.

This is an open access article licensed under the terms of the Creative Commons Attribution Non-Commercial License (http://creativecommons. org/licenses/ by-nc/3. 0/) which permits unrestricted, non-commercial use, distribution and reproduction in any medium, provided the work is properly cited. 